\title{
Using a Pedagogical Agent to Support Students Learning to Program
}

\section{Dylan Keifer Finch, Virginia Tech}

I am a Master's student researching computer science education and human-computer interaction at Virginia Tech.

\section{Prof. Stephen H Edwards, Virginia Tech}

Stephen H. Edwards is a Professor and the Associate Department Head for Undergraduate Studies in the Department of Computer Science at Virginia Tech, where he has been teaching since 1996. He received his B.S. in electrical engineering from Caltech, and M.S. and Ph.D. degrees in computer and information science from The Ohio State University. His research interests include computer science education, software testing, software engineering, and programming languages. He is the project lead for Web-CAT, the most widely used open-source automated grading system in the world. Web-CAT is known for allowing instructors to grade students based on how well they test their own code. In addition, his research group has produced a number of other open-source tools used in classrooms at many other institutions. Currently, he is researching innovative for giving feedback to students as they work on assignments to provide a more welcoming experience for students, recognizing the effort they put in and the accomplishments they make as they work on solutions, rather than simply looking at whether the student has finished what is required. The goals of his research are to strengthen growth mindset beliefs while encouraging deliberate practice, self-checking, and skill improvement as students work. 


\title{
Using a Pedagogical Agent to Supplement Limited Facility in Computer Science Education
}

\author{
Dylan Finch \\ Department of Computer Science \\ Virginia Tech \\ Blacksburg, VA, USA \\ dkfinch@vt.edu
}

\author{
Dr. Stephen Edwards \\ Department of Computer Science \\ Virginia Tech \\ Blacksburg, VA, USA \\ edwards@cs.vt.edu
}

\author{
Mukund Rajagopal \\ Department of Computer Science \\ Virginia Tech \\ Blacksburg, VA, USA \\ mrmukund@vt.edu
}

\begin{abstract}
Many colleges face a lack of support for their computer science students. There is not enough faculty to give each student adequate instructional and emotional support. This experience report describes the design, implementation, and evaluation of a solution to this problem: a pedagogical agent named Maria. A pedagogical agent is a human-like interface designed to simulate the relationship between teacher and student. In this way, Maria functions like a virtual teaching assistant, filling the gap left by inadequate human facility. Students can ask Maria natural language questions about computer science and get natural language responses. Maria was designed to: (1) make students want to ask her questions, (2) answer student questions, and (3) provide emotional support to students. Maria's implementation focuses on achieving these goals. To make students want to ask questions, Maria is relatable and easy to access. To make sure Maria was able to answer questions, she was programmed with the answers to many common computer science and general knowledge questions. She can also walk students through more complicated issues, like finding the cause of a NullPointerException. Finally, to provide emotional support to students, Maria will give students tips on how to improve their score on programming assignments and will congratulate students when they correctly fix problems with their code. Maria will be evaluated by examining usage to find out the proportion of students who asked questions, the proportion of students who used Maria multiple times, and whether students found Maria helpful.
\end{abstract}

\section{Introduction}

Computer science education is facing a crisis: a lack of adequate support for students. At one university, there are not enough instructors to provide each student with proper support. From 2009 to 2018, the number of undergraduate computer science students at this university increased from 294 to 875 , a $198 \%$ increase [1]. In the same time, the total number of faculty members working in the computer science department (including graduate students employed by the department) only increased from 135 to 255 (an 89\% increase) [2]. This university is not alone in the struggle to keep up with student growth, however. According to an article from the New York Times, the number of computer science majors doubled from 2013 to 2017, but the number of tenure-track professors only increased by $17 \%$ in the same time [3]. This lack of instructional support has negative impacts on students. As the number of students increases, the student to teacher ratio declines. Classes in some universities are taught in auditoriums with hundreds of students [4]. These large class sizes can make it harder for instructors to answer student questions and provide individualized help, resulting in a negative impact on student 
learning, when compared to smaller class sizes [5]. Teaching Assistants (TAs) can be a replacement for professors, but even their numbers are not enough to help every student. At our university, there are some days when it can take hours for a student to get a question answered by a TA. Some students will give up because the lines are too long, and many will not get effective one-on-one support because of the limited time a TA has to work with each student.

These problems are made worse by the fact that programming is a hard and discouraging skill to learn. One researcher suggested that it could take 10 years to fully understand how to program [6]. Another researcher found that about $71 \%$ of students in the field have negative emotions when learning to program for the first time [7]. Students who use automated grading systems (like Web-CAT) may get even more discouraged by the constant negative feedback of the system. The automated grader will list all the problems in a student's code without providing any encouragement. It has been shown that negative feedback like this can cause negative emotions and can negatively impact a person's belief in their ability to complete a task [8]. This can be detrimental in such a hard subject. Providing better emotional support could improve student attitudes and increase motivation to fix problems.

These problems could be solved by hiring more TAs and training them to support students emotionally, but this can be prohibitively expensive for large courses and the need for TAs fluctuates wildly from day to day. A better solution is something that is easily accessible, is available any time, can answer student questions, and can give emotional support: a virtual TA. This paper will explore the design, implementation, and evaluation of such a virtual TA.

\section{Scope and Limitations}

This experience report details the creation of a pedagogical agent [9] in the form of a chatbot [10] named Maria. Maria is accessible to students through an online system that grades student programming assignments, Web-CAT. When students submit a programming assignment, they can talk to Maria about their submission or errors in their code. Students can interact with Maria in three ways: (1) "Explain..." links which show up when the student has an error in their code (these allow the student to get an explanation of the error from Maria), (2) talking to Maria directly through a chat window, and (3) tips and encouragement that Maria will provide to students based on their latest code submission. When students talk to Maria through the chat window, they can ask natural language questions and Maria will respond with natural language answers.

The three primary goals of this project are: (1) students should want to use Maria, (2) Maria should answer student questions, and (3) Maria should provide emotional support. This paper will focus on designing, implementing and evaluating a system that meets these goals.

\section{Novelty}

Chatbots are an old technology, with the first use going back over 50 years [11]. They have also been applied to education [12] [13] [14] [15] and emotional support [16] [17] in the past, but these two use cases have never been combined. Maria also differs from previous educational 
chatbots with the goal of being able to answer any computer science question students have. Many chatbots are specialized to teach one topic, but Maria is designed to be flexible and to answer any question an introductory computer science student might have about programming.

\section{Background and Related Work}

\section{Chatbots and Related Tools}

A pedagogical agent is, simply, a chatbot used for education. And, a chatbot is "a computer program or an artificial intelligence which conducts a conversation via auditory or textual methods" [10]. The main goal of a chatbot is to speak with humans and sometimes offer advice, answer questions, or perform a service.

Chatbots have been used for a long time, for a variety of purposes. They were developed as early as in the 1960s [18]. Early chatbots were only passive beings. They could not retain information or offer real responses to what the user said. They could only try to keep the user talking by saying things like, "Go on," or, "Tell me more."

Today, chatbots are capable of much more than just passively listening to the user. Zo is one of today's most advanced chatbots [19]. Powered by machine learning, she can carry on a realistic conversation with the user. She even has her own Facebook profile, complete with personal information, posts, and a picture [20]. When speaking with Zo, the first thing you will notice is how she commands the conversation. Zo also has a consistent personality. All this helps the user to think of Zo as more of a real person, with a personality and "resulted in a more positive outlook, a feeling of emotional support, and a sense of social belonging" among users [21].

\section{AIML and ChatScript}

With the proliferation of chatbots, many tools have become available to help create chatbots. Specifically, AIML (Artificial Intelligence Markup Language) allows a chatbot designer to write out possible questions or comments from the user along with the corresponding chatbot responses. The system also allows for easily recognizing similar types of inputs ("What is AIML?" and "What does AIML mean?") and making the chatbot respond in the same way to either prompt [22]. In AIML, one user input and the output that it generates are together called a "rule."

A more recent tool, called ChatScript has built upon many of the ideas of AIML, making it even easier to create rules. ChatScript also supports longer back and forth exchanges between the user and the chatbot, called gambits. These gambits keep the chatbot's responses focused on the current topic [23].

\section{Web-CAT}

Web-CAT is an automatic grading system designed to process programming assignments [24]. The system is used at many universities. Computer science students upload code for 
programming assignments and Web-CAT will run tests on the code and give students feedback on whether the code compiles, whether it has any logical errors, and whether the student has written adequate tests for the code. Web-CAT will run reference tests on the code and then provide the student with feedback. This feedback is in the form of (sometimes hard to understand) compiler error messages or ambiguous test failure messages. This can be a problem, especially for newer programmers who are not yet familiar with all the terminology used in typical error messages. Web-CAT is also only focused on the student's score. If a student makes their code easier to read but it does not increase their score, Web-CAT does not care. This is bad for two reasons. First, it doesn't encourage students to be good programmers and, second, it can leave some students without recognition when they spend hours changing their code but do not increase their score.

Part of the goal of this project is to make Web-CAT more helpful and more beneficial to students. Web-CAT is extendable and when we created Maria, we built her into the existing Web-CAT grading system. This not only made it much easier for students to access our agent, but it also allowed us to use Maria to fix some of the problems present in Web-CAT. Maria will give students more detailed error messages and provide support for struggling students.

\section{Making it Easy to Learn}

Many factors can play a role in the way students learn. One of these factors is the similarity between the student and the teacher. It has been shown that students tend to learn better, when their teacher is like them [25]. Another factor that can play a role in education is empathy or emotional support from teachers to students. Education should be more than just the teacher reciting facts to the students. The teacher should be invested in the student and should show that they care. Social interaction can also play a role [26]. It can be much more valuable to learn something by discussing it than just copying notes from a board. We incorporated all these ideas into Maria to maximize the educational benefits of the system.

\section{Design Goals}

As described in the Introduction, the problem facing computer science education is a lack of support for students trying to learn the subject. Thus, when we began designing Maria, we created a set of design goals to make sure that Maria could solve this problem. These goals are: (1) students should want to use Maria, (2) Maria should answer student questions, and (3) Maria should provide emotional support. These goals are discussed more thoroughly in the following sections.

\section{Students Should Want to Use Maria}

This goal has two main parts: making sure Maria is easily accessible and making sure that students see Maria as knowledgeable, helpful, and supportive. 


\section{Maria Should Answer Student Questions}

Maria should be able to help these students by providing answers to their questions. If a student does not understand a concept or if they encounter an error, they should be able to ask Maria about it and Maria's answers should give the student a better understanding of it.

\section{Maria Should Provide Emotional Support}

Maria should provide encouraging messages to students who are struggling to make progress and provide congratulatory messages to students who are doing well. This would help to highlight the progress students make and to help them feel better about their work. It would also make them feel like they are not alone, even when TAs are not around to help.

\section{Implementation}

Maria was built using ChatScript. We created over 1,400 rules to match student questions to Maria's answers. For most of these rules, we wrote unique text to answer the student's question. Students can ask Maria questions about Java programming errors, Java language constructs (like loops and if statements), the use of types and methods in Java, programming concepts that go beyond Java (like abstraction and recursion), and testing (including information on how to set up tests and how to test certain parts of code).

Maria was built into the web interface of Web-CAT. The user interface for Maria was built using HTML and JavaScript. This JavaScript then communicates with a ChatScript server running Maria's conversational rules. When a student asks a question, it is sent to the ChatScript server which serves up Maria's response. Maria is accessible on the page that shows students the results of their last code submission. Students may click on a button in the corner of the page to open a chat window with Maria (see Figure 1). This chat window includes an animated head for Maria that will speak the answers aloud to the student, a chat history, and a text box for the user to ask a question (see Figure 2). Maria's answers are formatted with HTML, including links, lists, and code snippets.

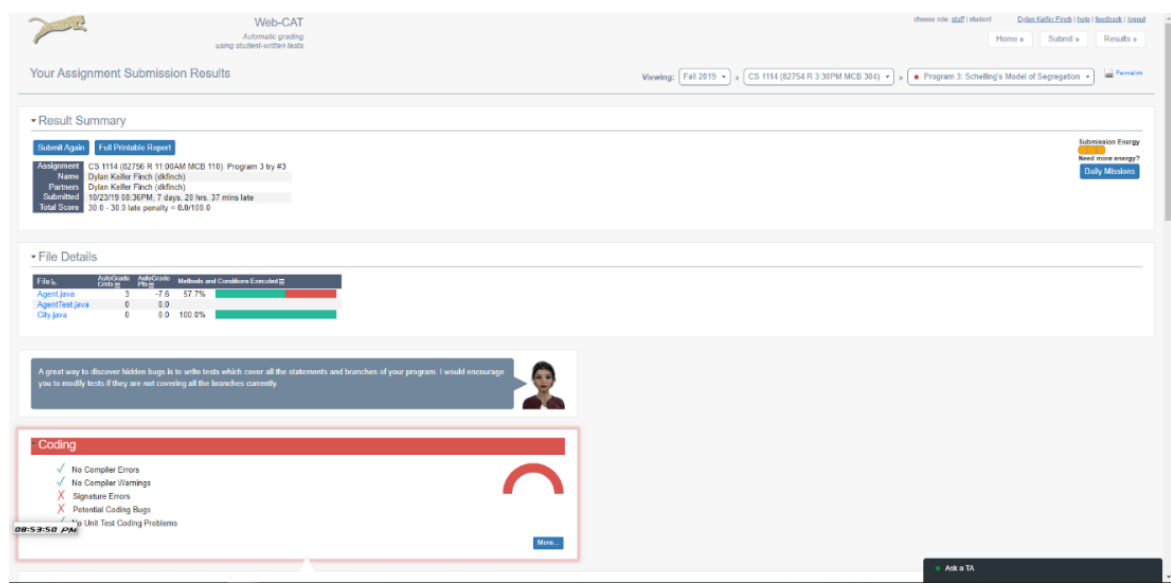

Figure 1. When students submit their work, Maria is accessible by clicking the "Ask a TA" button on the lower right of the page. 


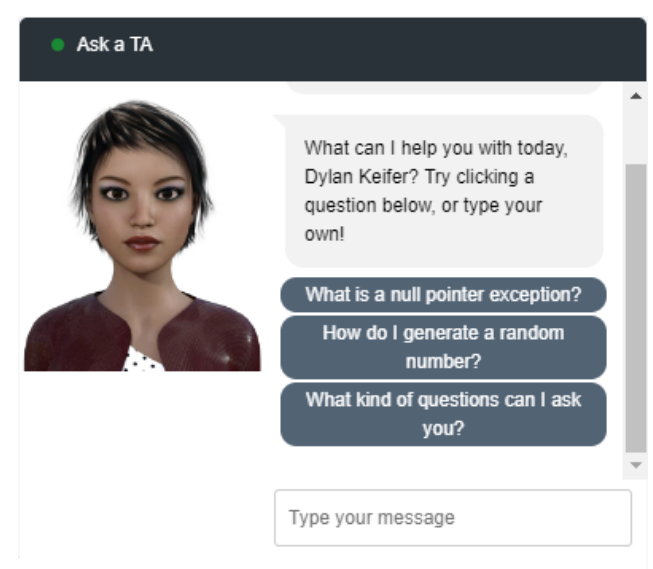

Figure 2. Maria's chat window.

Students can also interact with Maria using "Explain ..." links. When a student submits code that throws an error, Web-CAT will tell the student what kind of error occurred. Next to this, WebCAT will now also generate a link to explain the error (see Figure 3). If a student clicks the link, Maria will give them an explanation of when the error occurs and how they might be able to fix it. Currently, we have explanations for all Java exceptions, all Java syntax errors, and testing problems.

\section{Exceptions While Running Tests}

While running a reference test, your code encountered a NullPointerException on line 37 in Submission.java Explain..

Figure 3. An example of an "Explain ..." link.

The last way that students can interact with Maria is through feedback given by Maria. When a student submits their work to Web-CAT, Maria will occasionally give the student suggestions on how to make their code better (see Figure 1). These messages focus on readability and complexity. Some of the messages tell the students to reduce code complexity, by breaking up large methods or by reducing the number of if statements in the code. Others tell students to increase the number of comments in the code. Maria will also check students' code to see if they implement these suggestions and will then give the student kudos if they do.

Another key aspect of Maria is the use of Extended Dialogs. Extended Dialogs occur when the student asks Maria a question that cannot be answered in one short, concise response or when there may be multiple different answers, depending on the situation. When a student asks a complicated question like this, Maria will provide a short generic answer and then allow the user to ask Follow-Up Questions to clarify the meaning of the original question and get more specific answers. For example, if a student asks, "How do I write a test?" Maria will respond and ask the student to clarify what they are trying to test (A class? A method? An if statement?) and then provide a more specific answer based on what the student says. These Follow-Up Questions are pre-defined and shown as options to the user. The student can also type their own response if they would like to change the subject and stop asking Follow-Up Questions. Extended Dialogs 
and Follow-Up Questions make it so that students can be more relaxed with the phrasing of their questions and still find the information they are looking for. The first question they ask may not yield the answer they were looking for, but clarifications in Follow-Up Questions will eventually lead the student to the right answer.

For topics that are closely related, these Follow-Up Questions also allow students to find related information without having to type out another question that Maria might not understand. For example, if a student asks about how to test an if statement, they may follow up by asking about how to test a for loop.

One specific example of Extended Dialogs and Follow-Up Questions in action is the Extended Dialog we created to help students understand and debug NullPointerExceptions. If a student asks how they can fix a NullPointerException in Java, Maria will ask them a series of questions about where the error is occurring and will then provide possible solutions. If one solution does not work, Maria will suggest something else. Through Extended Dialogs and Follow-Up Questions, Maria can provide precise solutions to complicated problems.

Maria was also built with Conversation Starters. These are like Follow-Up Questions in that they are automatically generated responses for the student to pick from. However, they are shown at the beginning of an interaction with Maria, instead of in the middle of an interaction. These help to get a conversation started with a student who may not know what to ask. Conversation Starters include "What is a NullPointerException?", "How do I generate a random number?", and "What kind of questions can I ask you?" and can be seen above in Figure 2.

\section{Key Design Decisions}

This section describes the design decisions that were made to achieve the design goals. Each subsection corresponds to one design goal and lists the decisions made to support that goal.

\section{Students Should Use Maria}

Many studies have shown that people respond better to agents that are like themselves. Maria is female because one study found that female students respond better to female agents, with males not responding better to either sex [27]. Maria has a racially ambiguous skin tone and a name common across many cultures because universities often have many students of many different cultures and races and one study found that users respond better to agents that share a culture or race with themselves [28] [29]. Maria looks to be the age of an undergraduate student because one study suggested that students find undergraduate teaching assistants more helpful than graduate ones [30]. Making Maria younger also made her age closer to the age of the students who will be using her. These decisions have made the agent extremely relatable to students. The final design of Maria is shown above in Figure 2.

In addition to being relatable, we also created Maria to look and act competently. The clothes that Maria wears in her avatar (see Figure 2) make her look somewhat formal. Maria also has a 
strong base of knowledge, with some knowledge from outside of computer science. Together, these help students to trust that Maria is knowledgeable and will give correct answers.

Maria was also designed to be easy to access and easy to ask questions. Maria is built into WebCAT, which students already frequently use. From any page that shows grades, students can click a box and immediately ask Maria a question. To get a conversation started, Maria provides automatically generated Conversation Starters, so students do not have to think about how to phrase their first question. Once a student has started an interaction with Maria, some Follow-Up Questions are automatically generated and shown for the student to click on. This saves time for the student. These are also useful in cases where the student might not know how to continue the conversation with Maria or when they might not know how to formulate a question. To make using Maria even easier, "Explain ..." links are shown by any errors that Web-CAT finds in a student's code and allow the student to instantly go to an explanation of the error by Maria. All these functions make it very easy for students to access Maria and ask her questions, making it more likely students will use Maria.

Lastly, Maria was designed to be friendly. She will speak to students in an informative, yet friendly tone. She will have fun responses to some questions and can even play simple text-based games with the user. These fun interactions should make users want to interact with her more.

\section{Maria Should Answer Student Questions}

This goal focuses on making sure that Maria provides accurate and accessible answers to as many relevant questions as possible. To help achieve this goal, Maria was designed to provide answers to questions about every java error, about many Java language features, and about many general computer science topics. In total, Maria can answer over 1,400 questions. Maria was also designed to provide easily understandable answers. Most of the people using Maria will be students who are new to computer science or who are new to programming in Java. Because of this, and the fact that most compiler error messages are hard to understand for new programmers [31], Maria gives answers that use a minimal amount of complicated terminology.

To make sure that Maria can answer more complicated questions, we also created Extended Dialogs and Follow-Up Questions. This makes Maria able to answer more questions in more situations and makes interactions with Maria more comparable to human TAs.

\section{Maria Should Provide Emotional Support}

This goal focuses on making sure that students are motivated to continue working, even if they are having trouble getting their assignment score to increase. One way that Maria provides emotional support is through encouraging messages. Maria will recognize when a student is having trouble and will provide a message encouraging the student to keep working on that issue.

Maria will also provide congratulatory messages when a student is making progress on an assignment. An algorithm that detects effort put into code [32] will generate these messages. The algorithm will help to make sure that Maria correctly recognizes where a student made progress. 
The messages will also help to develop a growth mindset in students because the messages are not just tied to score and will appear when a student does something that is good practice.

\section{Evaluation}

To evaluate how effectively Maria was able to meet the design goals that were set out, the system was deployed to students in an introductory Computer Science class for the latter half of the Fall, 2019 semester. Students were not pressured to use Maria. They were simply told that she existed and that she could serve as an alternative to human TAs for students to have their questions answered. We recorded every time that a student had the opportunity to use Maria, every time a student opened the Maria chat window, every time a student asked Maria a question, and every time a student used one of the "Explain ..." links. Each time a student interacted with Maria, we recorded what the student said and how Maria responded. At the end of this semester, we also sent out a survey to students that asked for their opinions on the system.

\section{Results and Discussion}

\section{Raw Data}

From the time we released Maria to the end of the semester, we recorded 5,924 sessions where students had the opportunity to interact with Maria. A session is one visit to the page where Maria can be accessed. Of these 5,924 sessions, Maria was opened in 170 of them $(2.87 \%)$, an "Explain ..." link was used in 62 of them (1.05\%), and Maria was asked a question in 80 of them $(1.35 \%)$. In sessions where Maria was asked at least one question, Maria was asked an average of 3.09 questions.

The results can also be looked at on a per-student basis. From the time we released Maria to the end of the semester, we recorded 460 students who had the opportunity to interact with Maria. So, 460 students visited the page where Maria can be accessed. Of these 460 students, 122 students opened the Maria chat window (26.46\%), 46 students used an "Explain ..." link (9.98\%), and 62 students asked Maria a question (13.45\%). Students who asked Maria at least one question asked an average of 3.98 questions. Only 12 students asked Maria a question in more than one session.

In total, "Explain ..." links were used 92 times and students said something to Maria 247 times. This includes all questions asked by students and Follow-Up Questions suggested by Maria. Of the 247 user inputs given to Maria, 49 (19.84\%) were Conversation Starters suggested by Maria, 93 (37.65\%) were Follow-Up Questions suggested by Maria, and 105 (42.51\%) were nonsuggested, user-generated questions. All the suggested Conversation Starters and Follow-Up Questions are guaranteed to get appropriate answers from Maria. So, all the suggested inputs produced appropriate outputs. Of the 105 non-suggested inputs, 46 (43.81\%) were not answerable. These included random strings of letters and questions about topics not related to programming that Maria is not designed to answer. The other $59(56.19 \%)$ of the non-suggested inputs were answerable. Of the 59 answerable questions, Maria answered $8(13.56 \%)$ correctly 
and $51(86.44 \%)$ were answered incorrectly due to a lack of knowledge built into Maria (see Figure 4 below).

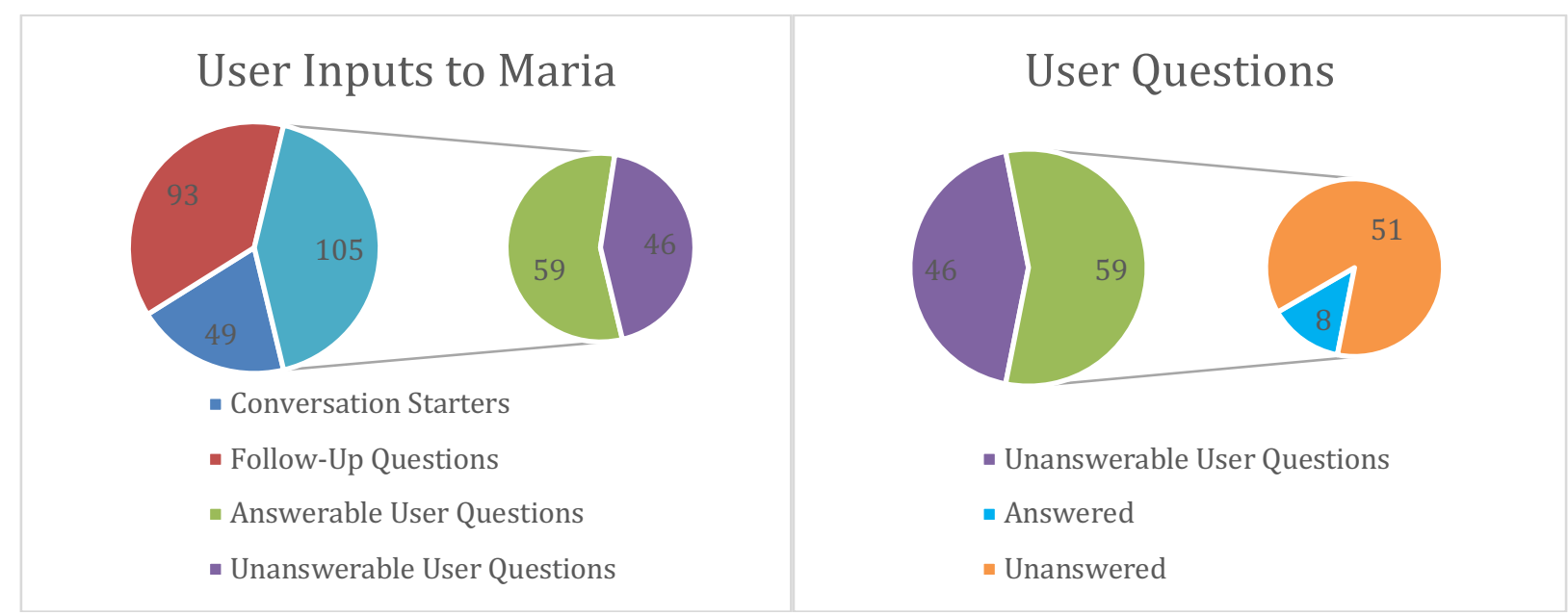

Figure 4. On the left is a pie chart showing how many questions were suggested by Maria (Conversation Starters and Follow-Up Questions) and how many were User Questions, questions that were not suggested by Maria. These User Questions are broken up into questions that Maria should have been able to answer and those she was not designed to answer. On the right, the Answerable Questions are further broken up into the questions that were answered correctly and those that were not.

\section{Discussion Related to Design Goals}

In the following subsections, we will discuss how we met or failed to meet the design goals set forth in a previous section of this paper.

\section{Students Should Want to Use Maria}

The data is encouraging. Of the 460 students, 122 interacted with Maria at least once. This is $26.46 \%$ of the students in the class. Almost one in four students had a need that they thought Maria could fill. This reinforces the idea that this is a problem that needs to be solved. Many students are sick of waiting in long TA lines and would be happy to use a system that could provide them with an instant answer to their questions.

Additionally, we saw great success with "Explain..." links, Conversation Starters, and FollowUp Questions. Of the 460 students, 46 used an "Explain..." link. As for Conversation Starters and Follow-Up Questions, they were the majority of the inputs that Maria received. This shows that students do want a way to easily ask questions and that ours seem to be working.

However, the data also tells us that we still have a lot of work to do. Only 62 of the 460 students asked Maria a question. And of these students, only 12 asked Maria a question in more than one session. This suggests that many students tried to use Maria once, were not happy with the results, and did not use her again. Maria was not able to answer most of the user-generated 
questions (see the next subsection). Also discouraging is the fact that of the 5,924 opportunities students had to interact with Maria, they only interacted with her in 170.

\section{Maria Should Answer Student Questions}

This is a definite weak point of the current implementation of Maria. Maria was able to answer many student questions. However, most of the questions that she answered were questions that she suggested asking. This includes Conversation Starters and Follow-Up Questions. In total, Maria received 339 user inputs. Of these, Maria was able to successfully provide answers to 92 "Explain ..." links, 49 Conversation Starters, and 93 Follow-Up Questions. Maria also received 59 answerable non-suggested, user-generated questions. Of these, Maria was only able to successfully answer 8 . The other 51 questions went unanswered simply because Maria did not have the knowledge to give an answer.

One of the longest series of Follow-Up Questions is an explanation of what a NullPointerException is and how to fix it. Students start by asking what a NullPointerException is. Maria will give them an explanation and then ask if they need help debugging the NullPointerException. If they answer yes, Maria will ask them a series of questions to try and figure out why the NullPointerException is happening. After asking these questions, Maria will provide suggestions on how the student can fix their code. This explanation has the largest number of Follow-Up Questions in all of Maria's rules. It is an excellent example of how Follow-Up Questions can be useful to students. Of all the students who asked Maria a question, 14 used this explanation. These 14 students asked an average of 5.64 Follow-Up Questions. Students are free to stop asking Follow-Up Questions at any time, so this is a very promising result. Students seem to like using the extended dialogs with Follow-Up Questions.

Student opinion surveys back up the fact that Maria's answering ability requires further development. On the whole, students believe that Maria's answers are not always useful, and the strongest responses were received to the question of whether students "want a virtual TA who is more capable than Maria", with $83 \%$ of students responding that they agree to some extent. At the same time, responses to this question also imply that students value the potential in Maria and do want to use Maria, if her responses are helpful and appropriate to the situation.

\section{Maria Should Provide Emotional Support}

In terms of providing emotional support, Maria's comments encouraging students or recognizing achievements have been described in earlier work [33]. By tracking measures of each submission by a student on one assignment and measuring differences between submissions, a suite of progress measures assesses different possible ways that the student's work may be progressing. Although the individual measures only indicate possible progress, presence of multiple indicators provides mutually supportive evidence of forward progress. When a submission possesses more than a minimum number of simultaneous measures, Maria will make a positive comment recognizing the student's efforts based on one of the triggered measures. Alternatively, if the measures do not indicate that a student is making progress, Maria will make an encouraging comment about something the student can focus on or continue working toward. The spirit of 
these comments is to recognize achievement where appropriate, while encouraging persistence and recognizing effort otherwise. As described in [33], Maria's comments are limited using a variable interval reinforcement schedule so that students do not receive more than approximately one such comment per hour. If comments appear on every submission, students can easily become blind to them and ignore them, but if they are not frequent enough, they may not have any effect.

\section{Summary of Discussion}

These results are promising for the future of this project. Many students are interested in using a system like this. Students seem to especially like Follow-Up Questions, Conversation Starters, and "Explain ..." links. The main issue with the current iteration of the system is the lack of knowledge. While Maria can answer over 1,400 questions, she needs to be able to answer even more to truly be useful to students.

\section{Improvements Made}

The evaluation showed that Maria was unable to answer 51 questions that she should have been able to answer. For each of these, a new answer was added. This means that Maria can respond to at least 51 more questions. (Additional answers were added that related to the unanswered questions and each newly added answer can be accessed by many phrasings of a question. So, more than 51 new questions can be answered.) Table 1 below shows some of the questions that she can now answer along with the new answer.

Table 1. This table shows some of the inputs that Maria could not respond to before the evaluation along with the new response that she will now give when given that input (or input like it).

\begin{tabular}{|l|l|}
\hline Question/Input & Maria's Response \\
\hline improper integer division & $\begin{array}{l}\text { In Java, when you divide two int variables, the result } \\
\text { will be a whole number. Java will not store the decimal } \\
\text { even if the numbers do not evenly divide. Java does } \\
\text { not round the numbers, it just throws away the } \\
\text { decimal. So, if we do 3/4, the result is 0.75 but Java } \\
\text { will throw away the decimal and just give us 0 as the } \\
\text { answer. This is called truncation. }\end{array}$ \\
\hline It tells me I haven't created any test cases but I did & $\begin{array}{l}\text { If have made a test but Web-CAT says you don't have } \\
\text { a test, this may be because you named the test class or } \\
\text { methods wrong. If you have a class called Thing, the } \\
\text { test class must be named Thing Test and if you want } \\
\text { to test a method called doStuff ( ), the test method } \\
\text { must be called testDosomething ( ) }\end{array}$ \\
\hline My name is [name] & $\begin{array}{l}\text { Hi, [name], my name is Maria. How can I help you } \\
\text { today? }\end{array}$ \\
\hline What is a Hashmap? & $\begin{array}{l}\text { A HashMap is a data structure that stores keys and } \\
\text { values. Each key is associated with one value and you } \\
\text { can use the key to get the value. If you've ever used } \\
\text { Python, they are basically dictionaries. }\end{array}$ \\
\hline
\end{tabular}




\begin{tabular}{|c|c|}
\hline How to use ternary operator & 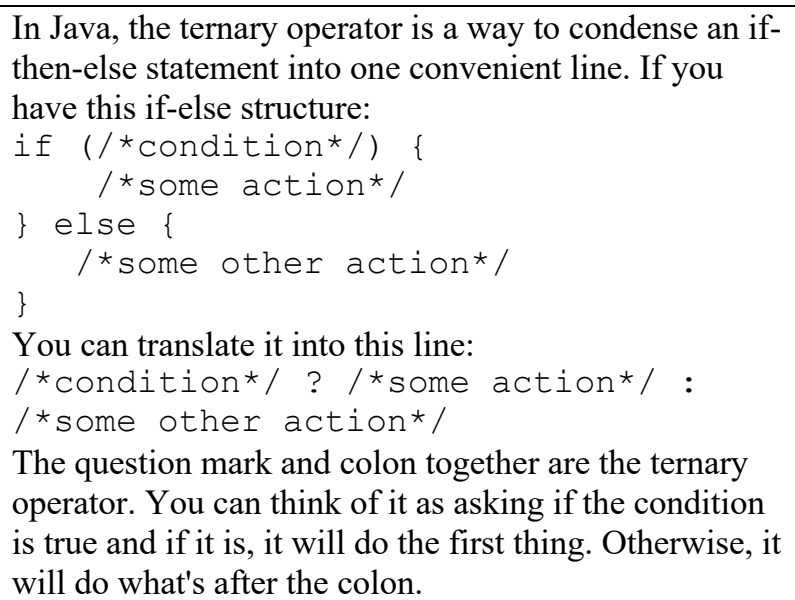 \\
\hline How do you test a constructor? & $\begin{array}{l}\text { To test a constructor, you simply have to call the } \\
\text { constructor somewhere in you test code. If the } \\
\text { constructor sets field values, you should probably } \\
\text { assert that the field values are set correctly. }\end{array}$ \\
\hline
\end{tabular}

\section{Future Work}

While Maria can currently respond to many questions, there are still many questions that she cannot respond to. Any future work on this project will surely include adding more questions and answers to Maria's knowledge base so that she can help in more situations. Along the same lines, it would also be helpful to add more Follow-Up Questions.

Two other areas of improvement would be the integration of a search engine, like Google, and the use of machine learning. A search engine could be used as a fall back if Maria does not know the answer to a certain question. She could simply search the internet and show the user a result which may answer their question. The use of machine learning could also be used to improve responses to every question. Right now, all questions and answers must be written by hand in advance. This process does not work for novel or rare questions where an answer has yet to be programmed in. A machine learning algorithm that could pull from a database of student questions, like a class forum, could be used to supplement hardcoded questions and answers. If this system could be fine-tuned enough, it would make the system very easy to scale and deploy to new fields (for which question and answers have yet to be written).

\section{Conclusion}

This paper described the design and implementation of a virtual TA named Maria. Maria was built into Web-CAT, an online automatic grading system for programming assignments, using HTML and JavaScript for the frontend and ChatScript for the backend.

Maria was designed with three design goals in mind: (1) students should want to use Maria, (2) Maria should answer student questions, and (3) Maria should provide emotional support. To support design goal 1, Maria was implemented to look relatable and component to students, to be easy to access, and to have a friendly personality. To support design goal 2, answers to over 1,400 questions were added to allow Maria to answer a wide range of questions, Extended 
Dialogs were added to allow Maria to give more complete answers to complex questions, and Follow-Up Questions were added to make it easier for students to access related knowledge. To support design goal 3, Maria is able to give encouraging messages to students, giving them praise for improving their code or suggestions on how they could improve their code.

Following Maria's implementation, an evaluation was conducted to see how well Maria was meeting each of her design goals. This showed that Maria was partially achieving all her design goals. With respect to design goal 1 , the evaluation revealed $26 \%$ of all students in the class interacted with Maria at least once, but far fewer students asked a question or used Maria more than once. Future versions of Maria will need to find ways to make it easier for more students to interact with Maria. With respect to design goal 2, many students used Extended Dialogs and Follow-Up Questions, however many user-generated questions went unanswered. Future versions of Maria need to focus on answering more questions. With respect to design goal 3, students seemed ambivalent about Maria's encouragement. Future versions will need to find ways to make students more invested in Maria's comments

Maria still needs more work to be a complete virtual TA. However, the work present in this paper shows the potential that she has, to help students. Students want and need a system like this, and with more work, it will be able to help them.

\section{References}

[1] Office of Institutional Research, "Headcounts By Major," Virginia Tech, [Online]. Available: https://www.ir.vt.edu/data/student/headcountsByMajor.html. [Accessed 2 January 2020].

[2] Office of Institutional Research, "Faculty \& Staff Data," Virginia Tech, [Online]. Available: https://www.ir.vt.edu/data/facultyStaff.html. [Accessed 1 January 2020].

[3] N. Singer, "The Hard Part of Computer Science? Getting Into Class," The New York Times, 24 January 2019. [Online]. Available: https://www.nytimes.com/2019/01/24/technology/computer-science-courses-college.html. [Accessed 2 January 2020].

[4] D. Dryfoos, "With large classes and waitlists, CompSci feels growing pains. But are those pains unique to Duke?," Duke Chronicle, 24 October 2017. [Online]. Available: https:/www.dukechronicle.com/article/2017/10/with-large-classes-and-waitlists-compscifeels-growing-pains-but-are-those-pains-unique-to-duke. [Accessed 1 January 2020].

[5] G. V. Glass and M. L. Smith, "Meta-analysis of research on class size and achievement," Educational evaluation and policy analysis, vol. 1, no. 1, pp. 2-16, 1979.

[6] L. E. Winslow, "Programming pedagogy - a psychological overview," SIGCSE Bull., p. 17-22, 1996.

[7] N. Bosch, S. D'Mello and C. Mills, "What emotions do novices experience during their first computer programming learning session?," in International Conference on Artificial Intelligence in Education, 2013. 
[8] E. J. Kim and K. R. Lee, "Effects of an examiner's positive and negative feedback on selfassessment of skill performance, emotional response, and self-efficacy in Korea: a quasiexperimental study," BMC medical education, vol. 19, no. 1, p. 142, 2019.

[9] Wikipedia contributors, "Pedagogical agent," Wikipedia, The Free Encyclopedia, 21 December 2019. [Online]. Available: https://en.wikipedia.org/wiki/Pedagogical_agent. [Accessed 2 January 2020].

[10] Wikipedia contributors, "Chatbot," Wikipedia, The Free Encyclopedia, 26 December 2019. [Online]. Available: https://en.wikipedia.org/wiki/Chatbot. [Accessed 2 January 2020].

[11] J. Weizenbaum and others, "ELIZA---a computer program for the study of natural language communication between man and machine," Communications of the ACM, vol. 9, pp. 36-45, 1966.

[12] D. Fossati, B. Di Eugenio, S. Ohlsson, C. Brown and L. Chen, "Data driven automatic feedback generation in the iList intelligent tutoring system," Technology, Instruction, Cognition and Learning, vol. 10, pp. 5-26, 2015.

[13] G. Biswas, K. Leelawong, D. Schwartz, N. Vye and The Vanderbilt Teachable Agents Group, "Learning by teaching: A new agent paradigm for educational software," Applied Artificial Intelligence, vol. 19, pp. 363-392, 2005.

[14] S. Brophy, G. Biswas, T. Katzlberger, J. Bransford and D. Schwartz, "Teachable agents: Combining insights from learning theory and computer science," in Artificial intelligence in education, 1999.

[15] F. Tanaka and S. Matsuzoe, "Children Teach a Care-receiving Robot to Promote Their Learning: Field Experiments in a Classroom for Vocabulary Learning," J. Hum.-Robot Interact., vol. 1, pp. 78-95, 72012.

[16] M. Lee, S. Ackermans, N. As, H. Chang, E. Lucas and W. IJsselsteijn, "Caring for Vincent: A Chatbot for Self-Compassion," in Proceedings of the 2019 CHI Conference on Human Factors in Computing Systems, 2019.

[17] K. K. Fitzpatrick, A. Darcy and M. Vierhile, "Delivering cognitive behavior therapy to young adults with symptoms of depression and anxiety using a fully automated conversational agent (Woebot): a randomized controlled trial," JMIR mental health, vol. 4, p. e19, 2017.

[18] G. Guzeldere and S. Franchi, "Dialogues with colorful "personalities" of early AI," Stanford Humanities Review, vol. 4, no. 2, pp. 161-169, 1995.

[19] "Let's talk about Zo," Microsoft, 2020. [Online]. Available: https:/www.zo.ai/. [Accessed 2 January 2020].

[20] "Zo," Facebook, [Online]. Available: https://www.facebook.com/zo/. [Accessed 2 January 2020].

[21] H.-Y. Shum, X.-d. He and D. Li, "From Eliza to XiaoIce: challenges and opportunities with social chatbots," Frontiers of Information Technology \& Electronic Engineering, vol. 19, no. 1, pp. 10-26, 2018.

[22] Wikipedia contributors, "AIML," Wikipedia, The Free Encyclopedia, 31 December 2019. [Online]. Available: https://en.wikipedia.org/wiki/AIML. [Accessed 2 January 2020]. 
[23] Wikipedia contributors, "ChatScript," Wikipedia, The Free Encyclopedia, 15 October 2019. [Online]. Available: https://en.wikipedia.org/wiki/ChatScript. [Accessed 2 January 2020].

[24] "What is Web-CAT?," Virginia Tech, [Online]. Available: http://webcat.org/projects/Web-CAT/. [Accessed 2 January 2020].

[25] S. Apostol, O. Şoica, L. Manasia and C. Ştefan, "Virtual Pedagogical Agents In The Context Of Virtual Learning Environments: Framework And Theoretical Models.," eLearning \& Software for Education, no. 2, 2013.

[26] L. S. Vygotsky, Mind in society: The development of higher psychological processes, Harvard university press, 1980.

[27] A. L. Baylor, "The design of motivational agents and avatars," Educational Technology Research and Development, vol. 59, no. 2, pp. 291-300, 2011.

[28] A. Bandura, Self-efficacy: The exercise of control, Macmillan, 1997.

[29] A. L. Baylor and Y. Kim, "Pedagogical agent design: The impact of agent realism, gender, ethnicity, and instructional role," in International conference on intelligent tutoring systems, 2004.

[30] E. Patitsas and P. Belleville, "What can we learn from quantitative teaching assistant evaluations?," in Proceedings of the Seventeenth Western Canadian Conference on Computing Education, 2012.

[31] M.-H. Nienaltowski, M. Pedroni and B. Meyer, "Compiler error messages: What can help novices?," ACM SIGCSE Bulletin, vol. 40, no. 1, pp. 168-172, 2008.

[32] S. Edwards and Z. Li, "Towards progress indicators for measuring student programming effort during solution development," in Proceedings of the 16th Koli Calling International Conference on Computing Education Research, 2016.

[33] S. H. Edwards and Z. Li, Designing boosters and recognition to promote a growth mindset in programming activites, ASEE, 2019.

[34] J. Allen, G. Ferguson and A. Stent, "An architecture for more realistic conversational systems," in Proceedings of the 6th international conference on Intelligent user interfaces, 2001. 